This is the post print version of the article, which has been published in European Journal of Clinical Nutrition. 2019, 73, 112-120. https://doi.org/10.1038/s41430-018-0267-y

\title{
Predictive value of the Mini Nutritional Assessment Short Form (MNA-SF) and Nutritional Risk Screening (NRS2002) in hip fracture
}

Authors: Heli Helminen, Tiina Luukkaala, Juha Saarnio \& Maria S. Nuotio

\section{Abstract}

Background/Objectives: To examine Mini Nutritional Assessment short form (MNA-SF) and Nutritional Risk Screening 2002 (NRS2002) as prognostic indicators of postoperative complications, length of hospital stay (LOS), readmissions, mobility, living arrangements and mortality after hip fracture.

Subjects/Methods: Population-based prospective data were collected on 265 consecutive hip fracture patients aged 65 and over. Nutritional status according to MNA-SF and NRS2002 was assessed on admission. Outcomes were postoperative complications, LOS, readmissions and mortality one and four months post fracture and changes in mobility level and living arrangements four months post fracture.

Results: At baseline 18 (7\%) patients were malnourished and $108(41 \%)$ at risk of malnutrition according to MNA-SF. According to NRS2002 11 (4\%) patients were at severe risk and 56 (21\%) patients at moderate risk of malnutrition. Only MNA-SF predicted mortality, LOS and readmissions. Both instruments proved ineffective in predicting changes in mobility level and living arrangements.

Conclusions: MNA-SF is superior to NRS2002 in predicting short-term hip fracture outcomes.

\section{Introduction}

The incidence of hip fracture is currently high and expected to increase due to population aging. Hip fracture is the most common serious injury in older people (1-2). There are many risk factors for falls, such as older age, unsteady gait, cognitive impairment and malnutrition (3). Malnutrition is common among older people; as many as half to two-thirds are at nutritional risk or malnourished (4-7). Lim et al. in their prospective cohort study on diagnosis-related groups (DRG) found that malnutrition led to longer hospital stay, more readmissions and mortality (8). In many studies malnutrition in hip fracture patients has been found to be associated with increased risk of perioperative complications, prolonged LOS, loss of mobility, a need for more assisted form of living arrangements and increased risk of mortality (6,7,9-11). Identifying patients likely to benefit from nutritional support could reduce morbidity and mortality, improve quality of life and reduce medical costs. Nutritional therapy restores and maintains the nutritional status of the patient and expedites recovery from trauma.

The European Society for Clinical Nutrition and Metabolism (ESPEN) guidelines of 2002 recommended different nutritional screening tools in different patients: NRS2002 for patients in hospital, MUST (Malnutrition Universal Screening Tool) for adults living in the community and MNA for older patients (12). In a review from 2010 Rasmussen et al. assessed three nutritional screening tools (NRS2002, MNA, MUST) for measuring nutritional risk in hospitals. Their opinion was that NRS2002 is best validated (13). Van Bokhorst-de van der Schuerin et al. evaluated 32 nutrition assessment methods in a comprehensive review article in 2014 (14). They found that there was no single instrument to screen nutritional status adequately and to predict poor nutrition related outcomes. For adults NRS2002, MUST and SGA (Subjective Global Assessment) performed well in half of the studies reviewed, but not for older patients. MNA performed fairly well to well in older patients. The authors recommended a comparison of different tools in different patient groups. 
The MNA test was validated in 1994 and subsequently used in hundreds of studies. It is a highly specific, reliable and validated screening instrument for older patients in various care settings (15). MNA-SF was developed in 2000 as a screening tool to assess nutritional status (16). It is simple, non-invasive, cheap and user-friendly. The NRS2002 test is a more recent screening instrument developed by an ESPEN working group. It classifies the severity of patients' diseases in combination with degrees of malnutrition. The test has one point correction for age $>70$ years $(12,17)$.

The aim of this study was to compare MNA-SF and NRS2002 screening tools as predictors of the short-term outcomes of postoperative complications, LOS, readmission, mobility, living arrangements and mortality in an unselected population-based sample of older hip fracture patients. Koren-Hakim et al. recently published an article comparing MNA-SF, NRS2002 and MUST in hip fracture patients and found that all three screening tools were adequate for assessing malnutrition, but only MNA-SF could predict readmission and mortality (18). The routine implementation for the nutritional assessment of hip fracture patients could have a significant medical and socio-economic impact.

Material and methods

Study population and design

The study includes all 265 consecutive patients over 65 in the Hospital District of Southern Ostrobothnia, Finland who suffered their first hip fracture between November 2015 and March 2017. Pathological or periprosthetic fractures were excluded. The population of the hospital district is approximately 200,000 and all hip fractures are treated in Seinäjoki Central Hospital, which is the only hospital in the district providing trauma surgery.

Of the patients $58 \%$ were operated on within 24 hours and $89 \%$ on within 48 hours of admission to our hospital. The patients were in the Central Hospital 5 days (mean) and after that in primary healthcare hospitals. The nurses on the orthopaedic ward were instructed to give daily nutritional supplements rich in energy ( $300 \mathrm{kcal})$ and protein $(20 \mathrm{~g})$ twice a day to all patients in addition to four meals enriched with energy and protein. It was advised to continue this in the primary care hospitals to which the patients were transferred for rehabilitation.

The baseline data were collected during the perioperative hospital stay on the orthopaedic ward mainly by a single geriatric nurse interviewing the patients or their representatives or hospital staff and using the medical records. MNA-SF and NRS2002 assessments were completed by the same geriatric nurse. Some of the patients (43\%) had an in-hospital comprehensive geriatric assessment and for these patients detailed geriatrician's instructions on care and rehabilitation, including nutritional supplementation, were sent in the discharge letter to the primary care hospitals after the fracture operation.

The follow-up data were collected from the same informants by telephone interviews at one and four months after surgery by the same geriatric nurse. The nurse moreover collected the data on LOS in the primary care hospitals, readmissions to the central hospital, complications and elicited the use of the nutritional supplements. The ethics committee of our hospital district approved the study design. All the patients or their representatives gave informed consent. 
Variables

MNA-SF consists of six sections: appetite or eating problem, recent weight loss, mobility impairment, acute illness/stress, dementia or depression and body mass index. Its scores are 0-7 points $=$ malnourished, 8-11 points $=$ at risk of malnutrition and 12-14 points $=$ normal nutritional status (16). To measure BMI, the patients' height and weight were noted as reported by the patients or caregivers or extracted from the patient files, and if not available, as estimated by the nurses on the orthopaedic ward.

NRS2002 is based on the assessment of severity of disease (mild, moderate or severe) and nutritional status by $\mathrm{BMI}$ or by weight loss in three months or by eating problem (17). Both categories give zero to three points and if a patient is over 70 years one more point is added. Hip fracture gives one point. NRS2002 scores are 0-2 points $=$ normal status $/$ mild malnutrition risk, 3-4 = moderate malnutrition risk and 5-7 = severe risk of malnutrition.

The preoperative American Society of Anesthesiologists (ASA) risk scores were used to assess general health at the time of the fracture. There are five classes: 1 ) healthy person, 2) mild systemic disease, 3 ) severe systemic disease, 4) severe systemic disease constituting is a constant threat to life, 5) a moribund person who is not expected to survive without the operation (19). Having a prefracture diagnosis of memory disorder was defined according to the national guideline set by a specialist in geriatric medicine or neurology (20).

Baseline independent mobility was defined as being able to walk independently without personal help. Living in an institution referred to residing in a primary care hospital or residential care home providing 24hour care. LOS refers to duration of stay in the central hospital and in the primary care hospital after the fracture combined. The cost of LOS is the price of the hospital stay on the ward in the central hospital (app. $1400 € / d$ ) and in the primary care hospital (app. 300€/d).

\section{Statistical analysis}

Differences in the distribution of age, gender, BMI, Alb (serum albumin), type of hip fracture, ASA scores, regular medications, diagnosis of memory disorder, mobility, living arrangements, time to operation and NRS2002 according to MNA-SF were analysed by independent samples Kruskall-Wallis test or Pearson's Chisquare test. Due to the skewed distributions, continuous variables were described by medians with ranges and modelled by nonparametric tests. The differences in the outcome variables between the categories of MNA-SF and NRS2002 were also tested using the abovementioned tests (Table 1 and 2).

The variability between MNA-SF and NRS2002 was shown using the Bland- Altman plot. The values of the measurements were first normalized by reflecting and taking logarithm (Ln).

Multivariable multinomial logistic regression analyses with odds ratios (OR) and $95 \%$ confidence intervals (Cl) were conducted to examine associations of MNA-SF and NRS2002 with changes in mobility and living arrangements. The analyses were adjusted for age, sex, ASA grade and fracture type. Multivariable Cox proportional hazards models with hazard ratios (HR) and $95 \% \mathrm{Cls}$ were conducted to examine the association of MNA-SF and NRS2002 with four-month mortality. The results of the mortality analyses were illustrated by survival curves. The models were adjusted for age, sex, ASA grade, fracture type, mobility 
level and living arrangements at baseline. Statistical analyses were carried out with SPSS (SPSS Inc., IBM Corp., Armonk, NY, USA), Version 24.0. The p-value $<0.05$ was considered statistically significant.

Results

Distributions of the basic characteristics at baseline between normal, those at risk of malnutrition and the malnourished according to MNA-SF are described in Table 1. At the time of the fracture $18(7 \%)$ subjects were malnourished, $108(40 \%)$ were at risk of malnutrition and $139(53 \%)$ of the patients had normal nutritional status according to MNA-SF. According to NRS2002 11 (4\%) subjects had severe, 56 (21\%) moderate and 198 (75\%) mild impaired or normal nutritional status. Symmetric measure Kappa-value (0.288) indicates a fair agreement between these two measurements (Fig 1).

Of the 265 patients $20(8 \%)$ had died in one month and $48(18 \%)$ in four months after the hip fracture. Especially patients who were malnourished according to MNA-SF had a poor prognosis. No NRS2002 category significantly predicted mortality (Fig 2, Fig 3, Table 3).

Mean LOS in the central hospital was five days (range 2-17days) in all nutritional groups assessed by MNASF and NRS2002. Including the stay in the primary care hospital, median LOS was 24 days (range 5-230) in malnourished patients, 22 days (3-159) in nutritional risk patients and 22 days (4-100) in normal nutritional status patients according to MNA-SF. The same figures according to NRS2002 were 22 days (range 5-114) in severe, 23 days (5-230) in moderate and 22 days (3-135) in normal to mild nutritional risk patients.

Duration of treatment and rehabilitation in the primary care hospital were longer for malnourished patients according MNA-SF but this was not statistically significant. Many malnourished patients died, which shortened LOS. The mean cost of LOS was 19,900€ for malnourished patients and 15,100€ for normal patients according to MNA-SF.

Of the patients $38(14 \%)$ were readmitted to the emergency department of the central hospital within one month and $57(22 \%)$ in the next three months after the fracture. Of the patients eight (3\%) had more than one readmission within one month and $23(9 \%)$ within four months. Malnourished patients as assessed by MNA-SF had significantly more readmissions than the other patients (Table 2). This means more complications in malnourished patients. Infections (wound, urinary, pulmonary, abdominal) were the most common reasons for readmissions (29\%) and new falls (17\%) the second.

Neither of the tests by multivariate multinomial logistic regression analysis predicted a change in living arrangements after four months. Risk of malnutrition according to MNA-SF (OR 1.63; Cl 0.86-3.07) and moderate risk of malnutrition according to NRS2002 (OR 2.20; $\mathrm{Cl} 1.07-4.51$ ) would seem to predict poorer mobility after four months (Fig 3,).

Of the patients 103 (43\%) were on nutritional supplements one month, but only $43(21 \%)$ four months after the operation. If the geriatrician had diagnosed malnutrition and instructed the primary care hospitals, the use of nutritional supplementation was significantly more common than without in-hospital geriatrician's recommendation ( $50 \%$ vs. $36 \% \mathrm{p}=0.036$ ). The use of nutritional supplements did not seem to affect infections or other complications. 


\section{Discussion}

Our findings demonstrate that hip fracture patients' nutritional status influences outcomes like mortality, LOS, readmissions and complications. Poor outcomes and death particularly concern the malnourished group. According to our observations, MNA-SF significantly predicted most of the outcomes while NRS2002 detected patients' nutritional risk inadequately and predicted outcomes poorly.

The prevalence of nutritional risk in our population of hip fracture patients ranged $25-47 \%$ depending on the nutrition screening tool used. MNA-SF identified more patients at risk of malnutrition or malnourished than NRS2002 identified moderate or severe risk of malnutrition. Similar results have been reported earlier $(18,21,22)$. On the other hand Baek et al. found that MNA-SF overestimated the nutritional risk in the elderly (23). Nevertheless, it may be more harmful to underestimate the nutritional risk than to overestimate it, especially in acute trauma patients.

In our study ASA scores were higher in malnourished patients. This indicates the patient's overall condition and high scores predict a greater number of comorbidities and mortality after hip fracture treatment (24). Over half of the malnourished patients had memory disorder but only $22 \%$ of the patients with normal nutritional status, thereby corroborating earlier reports $(5,11)$.

In our study patients malnourished according to MNA-SF had longer LOS (mean 48 vs. 27 days) than did the other patients. In our study LOS included total duration of stay in the central hospital and in the primary care hospital combined. NRS2002 did not reveal the same difference between nutritional groups. In the study by Koren-Hakim et al. mean LOS was 11 days, which probably refers to duration of stay on the surgical ward (in our study this was five days). These workers found no differences in LOS according to any nutritional screening tool - MNA-SF, NRS2002 and MUST (18). Their study was retrospective and the patients were from the period 2007-2009. Surgical treatments as well as postoperative care develop constantly, which may have an effect on rehabilitation time on a surgical ward. In their retrospective study Nicholson et al. assessed nutritional status using serum albumin and found that patients with low serum albumin had prolonged hospital stay (7). In our study serum albumin was likewise significantly lower in malnourished patients according to MNA-SF than in other groups. Like us, Drevet et al. confirmed that malnutrition measured by MNA was associated with longer LOS (5).

The number of readmissions among our patients was not high, but the majority of those who returned to the hospital were malnourished according to MNA-SF. Readmissions means complications or other problems in rehabilitation. No significant relationship with readmissions was observed between patients' nutritional status according to NRS2002. Olofsson et al. have also demonstrated that low MNA scores were associated with postoperative complications (25) while Karen-Hakim et al. observed no complications predicted by nutritional assessment tools (18).

MNA-SF predicted mortality well in our study. Respectively one third and over half (56\%) of the malnourished patients died one and four month after the hip fracture. Of the patients at risk of malnutrition and with normal nutritional status respectively $21 \%$ and $10 \%$ died within four months. Similar results have been reported earlier $(10,11,18,26)$. No significant difference could be seen between groups when using NRS2002.

It was recommended that meals rich in energy and protein and daily nutritional supplements should continue in the primary care hospitals, but this was only partially realized. Interestingly, the use of 
nutritional supplementation was more common among patients assessed by a geriatrician during acute hospital stay. This emphasizes the significant role of the physician, in addition to the nursing staff, in diagnosing and actively addressing nutritional problems. Botella-Carretero et al. observed that hip fracture patients taking energy-protein supplements showed better recovery of plasma proteins and suffered fewer postoperative complications (27). Jie et al. also found the benefit of nutritional support in patients at nutritional risk (28). The systemic review and meta-analysis by Cawood et al. provided evidence that high protein supplements produce clinical benefits such as reduced complications and readmissions (29). We observed no such benefit in our study, probably because too few patients were given the nutritional supplements. On the other hand a Cochrane review of 2016 concluded that there is low-quality evidence that oral multinutrient supplements may prevent complications after hip fracture, and no clear effect on mortality has been observed (30).

In addition to nutritional supplementation products, attention should be paid to overall sufficient protein and energy intake even in the cases of nutritional status assessed as normal by means of the screening tools, especially in high-risk patients such as older hip fracture patients. This has been emphasized in our recent report (31), where a substantial proportion of hip fracture patients with normal nutritional status assessed by the MNA-SF at the time of the fracture were prone to develop poor nutritional status during the next few months post-fracture. In another report by Jyväkorpi et al. high proportions of older people with normal nutritional status according to the MNA had poor protein intake and low diet quality (32).

Why MNA-SF does seem to predict outcomes better than NRS2002 in this patient group? It is likely that MNA-SF recognizes frailty better than NRS2002, which mainly includes comorbid conditions, by taking account of impaired mobility and neuropsychological problems. Besides malnutrition, these domains are well known to be among the most significant predictors of poor outcomes in hip fracture patients (10). Moreover, as observed in an earlier study, poor nutritional status measured by MNA-SF was found in a comprehensive geriatric outpatient assessment to be associated with impaired cognition, depressed mood, impaired mobility and grip strength six months after hip fracture (31). The superiority of the MNA-SF over NRS2002 in predicting outcomes in older hip fracture patients highlights the importance of a comprehensive rehabilitative approach when designing nutritional interventions for this extremely vulnerable group of patients.

The strengths of this study include new, relatively large and representative population-based material derived from real-life circumstances and prospective and consecutive design. The systemic data collection including MNA-SF, NRS2002 and follow-up data was mainly carried out by a single nurse, which enhances the reliability of the data. Another strength was that we were able to include a variety of the most significant outcome parameters in our study. Further, patients were not excluded from the investigation on the basis of any comorbidity or being institutionalized.

Our study also has some limitations. Hip fracture patients' weight and height are difficult to measure in the perioperative period because of pain. These were estimated by nurse. We focused on testing only the two nutritional assessment tools most used in our hospital. Moreover we recorded no specific comorbid diagnoses but only used ASA as an indicator of comorbidity. Finally, due to the non-interventional and observational nature of our study, the possible effect of the nutritional supplementation on the outcomes could not be reliably examined. 
It is important to recognize the risk of malnutrition, to prevent the deterioration of the nutritional state and to treat malnutrition. If nutritional supplements can prevent any complication and prolonged hospitalization they will be valuable both in economic terms and in terms of human well-being.

\section{Conclusion}

A screening tool must be cheap, simple and fast to administer and sensitive enough to detect patients at nutritional risk. MNA-SF seems to work better than NRS2002 for predicting short-term outcomes in hip fracture patients. Our findings suggest that NRS2002 does not take sufficient account of the deteriorated functional capacity and mental health associated with poor nutritional status in older hip fracture patients, typically characterizing the frail and multimorbid geriatric patient. More studies are needed to investigate if similar findings also apply to other groups of acutely hospitalized older patients.

\section{Conflict of interest}

The authors declare no conflict of interest.

\section{Acknowledgements}

Ms Kaisu Haanpää, RN, is gratefully acknowledged for her expert collection of the data and performing the MNAs on the patients. This study was financially supported by the State Research Financing of Seinäjoki Central Hospital.

\section{References}

1) Cheng S, Levy A, Lefaivre K, Guy P, Kuramoto L, Sobolev B. Geographic trends in incidence of hip fractures: a comprehensive literature review. Osteoporosis Int 2011; 22: 2575-86

2) Kanis J, Oden A, McCloskey E, Johansson H, Wahl D, Cooper C. A systemic review of hip fracture incidence and probability of fracture worldwide. Osteoporosis Int 2012; 23: 2239-56

3) Stolee P, Poss J, Cook R, Byrne K, Hirdes J. Risk factors for hip fracture in older home care clients. J Gerontol A Biol Sci Med Sci; 2009; 64: 403-10

4) Kaiser M, Bauer J, Rämsch C, Uter W, Guigoz Y, Cederholm T et al. Frequency of malnutrition in older adults: a multinational perspective using the mini nutritional assessment. J Am Geriatr Soc 2010; 58: 1734-8

5) Drevet s, Bioteau C, Maziere S, Couturier P, Merloz P, Tonetti J, Gavazzi G. Prevalence of proteinenergy malnutrition in hospital patients over 75 years of age admitted for hip fracture. Orthop Trauma Surg Res 2014; 100: 669-74

6) Correia M, Hegazi R, Higashiguchi T, Michel J, Reddy B, Tappenden K et al. Evidence-Based Recommendations for Addressing Malnutrition in Health Care: An Updated Strategy from the feed M.E. Global Study Group. Jamda 2014; 15: 544-50 
7) Nicholson J, Dowrick A, Liew S. Nutritional status and short-term outcome of hip arthroplasty. J Orthop Surg 2012; 20: 331-5

8) Lim S, Ong K, Chan Y, Loke W, Ferguson M, Daniels L. Malnutrition and its impact on cost of hospitalization, length of stay, readmission and 3-year mortality. Clin Nutr 2012; 31: 345-50

9) Carpintero P, Caeiro J, Carpintero R, Morales A, Silva S, Mesa M. Complications of hip fractures: A review. World J Orthop 2014; 18: 402-11

10) Nuotio $M$, Tuominen $P$, Luukkaala T. Association of nutritional status as measured by the MiniNutritional Assessment Short Form with changes in mobility, institutionalization and death after hip fracture. Eur J Clin Nutr 2016; 70: 393-8

11) Helminen $\mathrm{H}$, Luukkaala T, Saarnio J, Nuotio M. Comparison of the Mini-Nutritional Assessment short and long form and serum albumin as prognostic indicators of hip fracture outcomes. Injury 2017; 48: 903-8

12) Kondrup J, Allison S, Elia M, Vellas B, Plauth M. ESPEN Guidelines for Nutrition Screening 2002. Clin Nutr 2003; 22 (4): 415-21

13) Rasmussen $\mathrm{H}$, Holst M, Kondrup J. Measuring risk in hospitals. Clin Epidemiol 2010; 2: 209-16

14) van Bokhorst-de van der Schueren $M$, Guaitoli $P$, Jansma $E$, de Vet $H$. Nutrition screening tools: Does one size fit all? A systemic review of screening tools for the hospital setting. Clin Nutr 2014; 33: $39-58$

15) Vellas $B$, Villars $H$, Abellan $G$, Soto $M$, Rolland $Y$, Guigoz $Y$. Overview of the MNA-Its history and challenges. J Nutr Health Aging 2006; 10: 456-63

16) Rubenstein $L$, Harker $j$, Salva $A$, Guigoz $Y$, Vellas B. Screening for undernutrition in geriatric practice: developing the short-form mini-nutritional assessment (MNA-SF). J Gerontol a Biol Med Sci 2001; 56: 366-72

17) Kondrup J, Rasmussen H, Hamberg O, Stanga Z; Ad Hoc ESPEN Working Group. Nutritional risk screening (NRS2002): a new method based on an analysis of controlled clinical trials. Clin Nutr 2003; 22(3): 321-36

18) Koren-Hakim T, Weiss A, Hershkovitz A, Otzrateni I, Anbar R, Gross Nevo R et al. Comparing the adequacy of the MNA-SF, NRS-2002 and MUST nutritional tools in assessing malnutrition in hip fracture operated elderly patients. Clin Nutr 2016; 35: 1053-8

19) Sankar A, Johnson S, Beattle W, Tait G, Wijeysundera D. Reliability of the American Society of Anesthesiologists physical status scale in clinical practice. Br J Anaesth 2014; 113: 424-32

20) Suhonen J, Rahkonen $T$, Juva K, Pitkälä K, Voutilainen $P$, Erkinjuntti T. The treatment pathway of a memory disorder patient. Duodecim 2011; 127: 1107-16

21) Zhou J, Wang $M$, Wang $H$, Chi Q. Comparison of two nutrition assessment tools in surgical elderly inpatients in Northern China. Nutr J 2015; 14: 68

22) Holst M, Yifter-Lindgren E, Surowiak M, Nielsen $K$, Mowe $M$, Carlsson $M$ et al. nutritional screening and risk factors in elderly hospitalized patients: association to clinical outcome? Scand J Caring Sci 2013; 27: 953-961

23) Baek M, Heo Y. Evaluation of the efficacy of nutritional screening tools to predict malnutrition in the elderly at a geriatric care hospital. Nutr Res Pract 2015; 9: 637-43

24) Bjorgul K, Novicoff W, Saleh K. American Society of Anesthesiologist Physical Status score may be used as a comorbidity index in hip fracture surgery. J Arthoplasty 2010; 25: 134-7

25) Olofsson B, Stenvall M, Lundström M, Svensson O, Gustafson Y. Malnutrition in hip fracture patients: an intervention study. J Clin Nurs 2007; 16: 2027-38 
26) Bell J, Bauer J, Capra S, Pulle R. Concurrent and predictive evaluation of malnutrition diagnostic measures in hip fracture inpatients: a diagnostic accuracy study. Eur J Clin Nutr 2014; 68: 358-62

27) Botella-Carretero J, Iglesias B, Balsa J, Arrieta F, Zamarron I, Vazquez C. Perioperative oral nutritional supplements in normally or mildly undernourished geriatric patients submitted to surgery for hip fracture: a randomized clinical trial. Clin Nutr 2010; 29: 574-9

28) Jie B, Jiang Z, Nolan M, Efron D, Zhu S, Yo K, Kondrup J. Impact of nutritional support on clinical outcome in patients at nutritional risk: a multicenter, prospective cohort study in Baltimore and Beijing teaching hospitals. Nutrition 2010; 26: 1088-93

29) Cawood A, Elia M, Stratton R. Systemic review and meta-analysis of the effects of high protein oral nutritional supplements. Ageing Res Rev 2012; 11: 278-96

30) Avenell A, Smith T, Curtain J, Mak J, Myint P. Nutritional supplementation for hip fracture aftercare in older people. Cochrane database Syst Rev 2016; 30: 11:CD001880

31) Helminen $H$, Luukkaala T, Saarnio J, Nuotio M. Changes in nutritional status and associated factors in a geriatric post-hip fracture assessment. Eur Ger Med 2017; 8: 134-9

32) Jyväkorpi S, Pitkälä K, Puranen T, Björkman M, Kautiainen H, Strandberg T et al. High Proportions of older people with normal nutritional status have poor protein intake and low diet quality. Arch Gerontol Geriatr 2016; 67: 40-5

Title / Legend

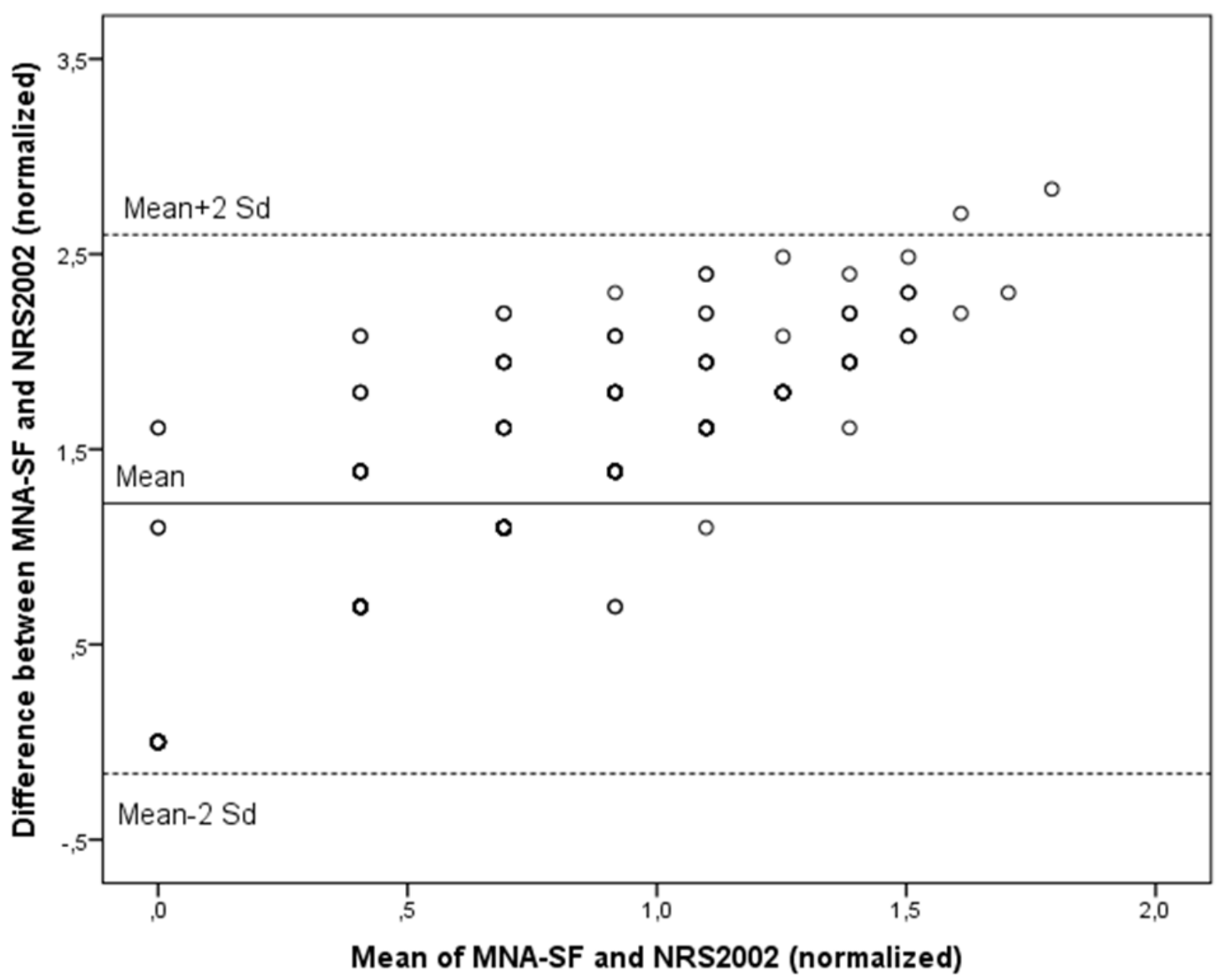

Fig 1. Bland-Altman plot of MNA-SF and NRS2002 ( $\mathrm{N}=265)$. 


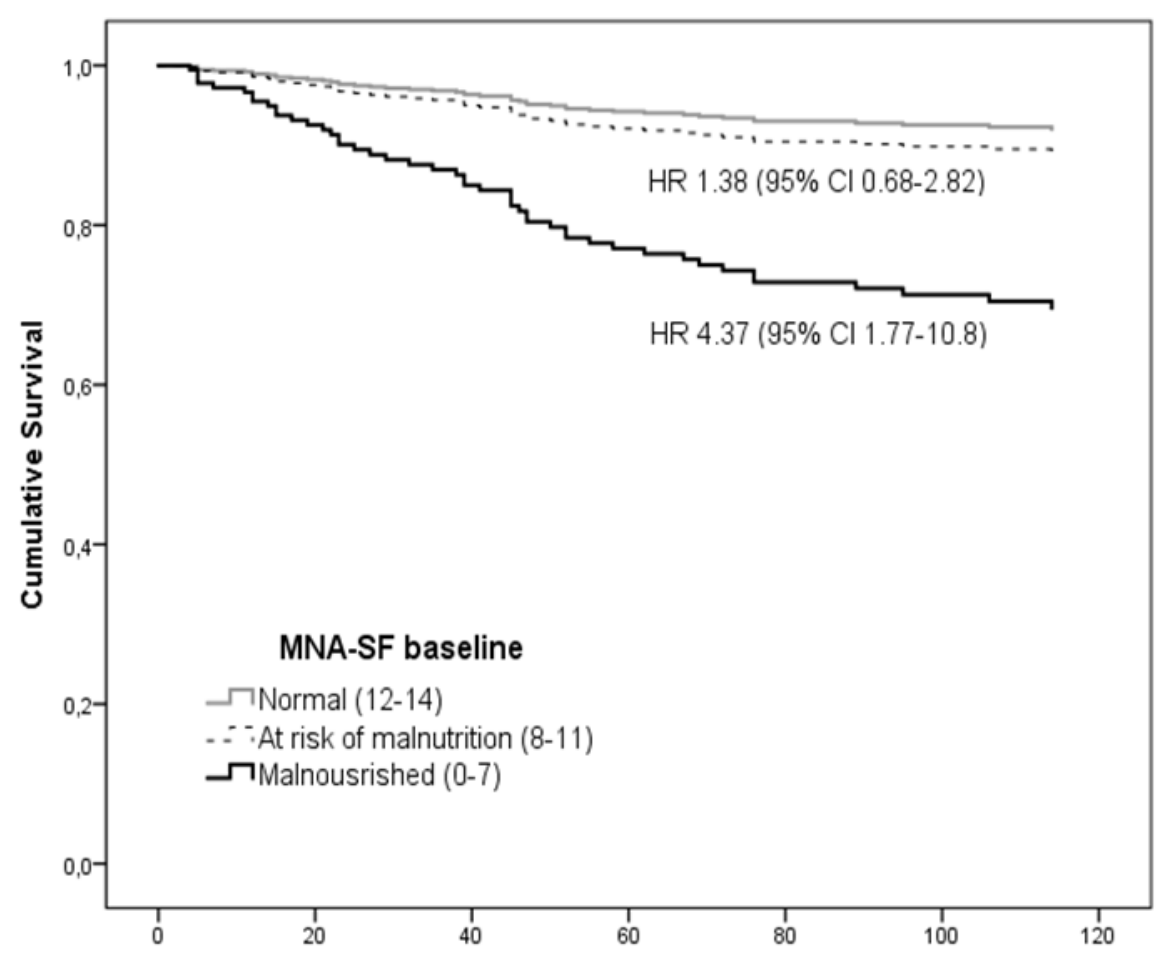

TIme (Days) from hip-fracture to the death or to the end of follow-up

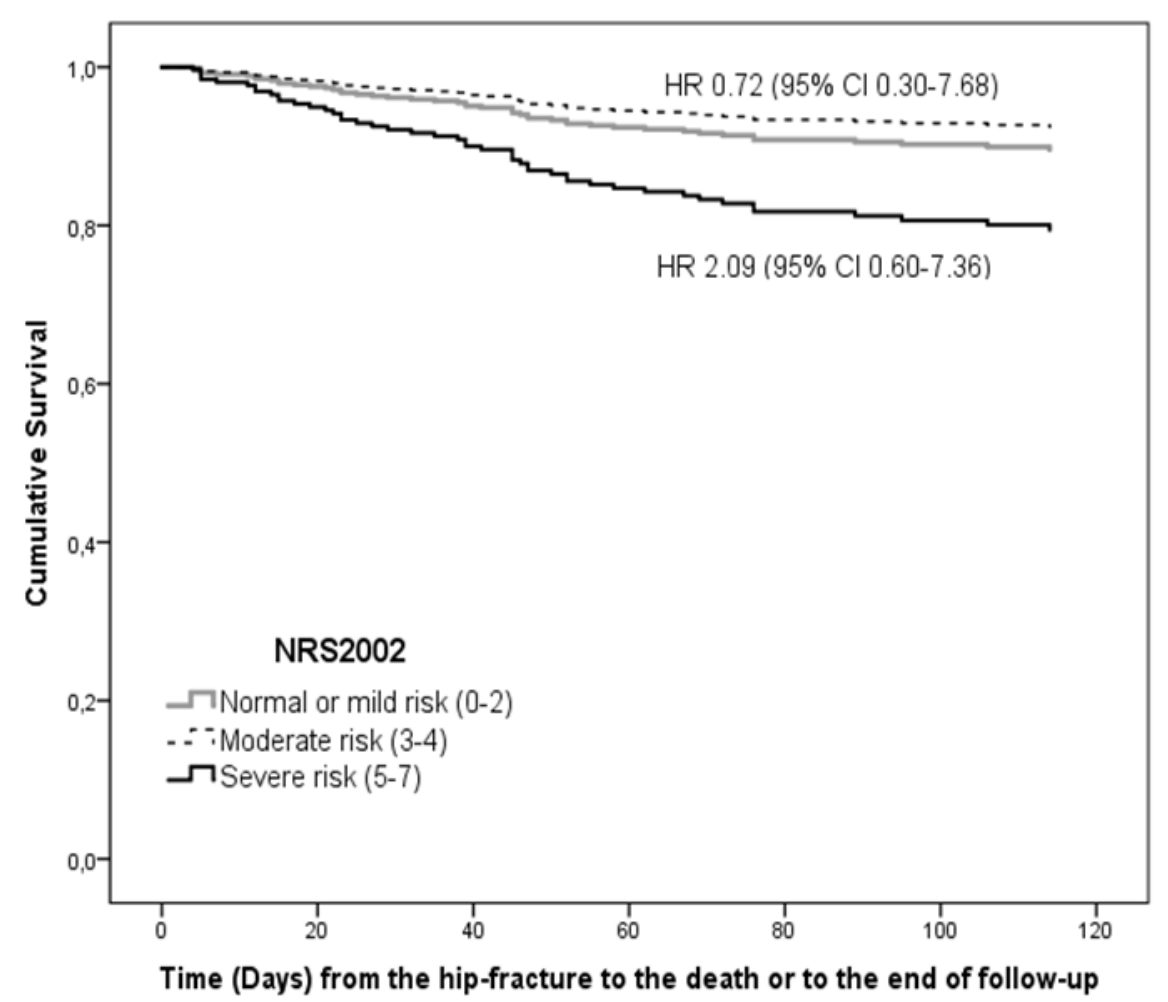

Fig 2. Four-month survival according to MNA-SF and NRS2002 ( $N=265)$.

Legend: Models were adjusted for age, sex, ASA and fracture type, moving and living arrangements at baseline. 

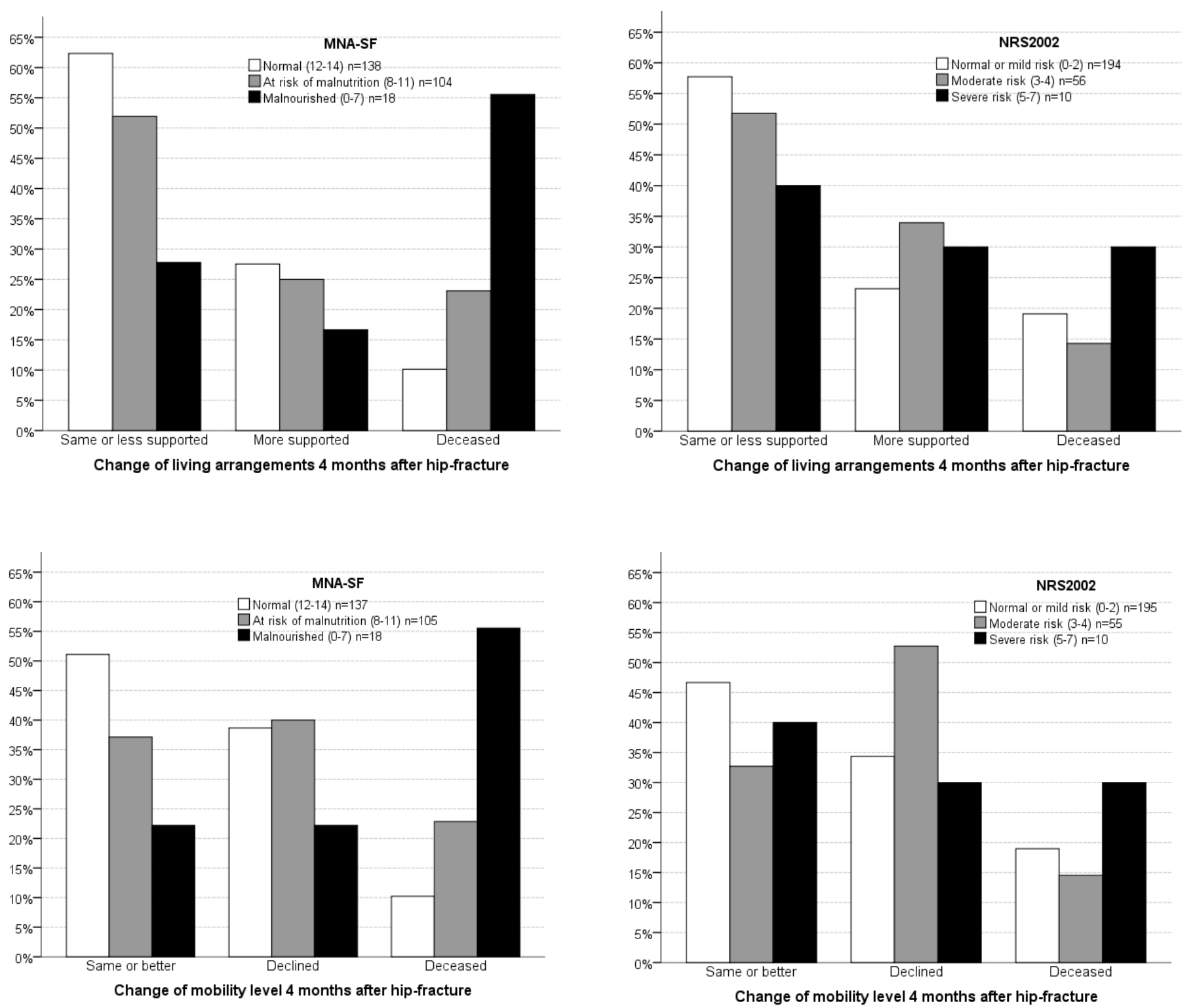

Fig 3. Changes in living arrangements and mobility level four months after hip-fracture according to MNASF and NRS2002 ( $N=260)$. 
Table 1. Distribution of basic baseline characteristics according to MNA-SF $(\mathrm{N}=265)$

\begin{tabular}{|c|c|c|c|c|c|c|c|}
\hline \multirow[b]{2}{*}{ Age, median (IQR, range) } & \multicolumn{2}{|r|}{$\begin{array}{c}\text { Normal } \\
\mathrm{n}=139(53 \%)\end{array}$} & \multicolumn{2}{|c|}{$\begin{array}{l}\text { Risk of malnutrition } \\
n=108(40 \%)\end{array}$} & \multicolumn{2}{|c|}{$\begin{array}{c}\text { Malnourished } \\
n=18(7 \%)\end{array}$} & \multirow{2}{*}{$\begin{array}{l}p \\
0.427\end{array}$} \\
\hline & 84 & $(78-90 ; 50-103)$ & 85 & $(79-91 ; 65-102)$ & 87 & $(81-93 ; 69-98)$ & \\
\hline Women, n (\%) & 91 & $(66)$ & 73 & (67) & 13 & (72) & 0.836 \\
\hline BMI, median (IQR, range) & 25 & $(23-28 ; 19-57)$ & 23 & $(21-26 ; 16-37)$ & 21 & $(18-24 ; 18-31)$ & $<0.001$ \\
\hline Alb, median (IQR, range) & 35 & $(32-37 ; 26-43)$ & 34 & $(31-37 ; 22-43)$ & 32 & $(28-35 ; 22-38)$ & 0.001 \\
\hline Fracture type, n (\%) & & & & & & & 0.357 \\
\hline Neck of femur & 88 & (63) & 62 & (57) & 14 & (78) & \\
\hline Intertrochanteric & 41 & (30) & 40 & (37) & 4 & (22) & \\
\hline Subtrochanteric & 10 & (7) & 6 & (6) & 0 & (0) & \\
\hline ASA, n (\%) & & & & & & & 0.025 \\
\hline $1-2$ & 24 & (18) & 10 & $(10)$ & 1 & (6) & \\
\hline 3 & 89 & $(66)$ & 77 & (73) & 9 & (53) & \\
\hline $4-5$ & 21 & $(16)$ & 19 & (17) & 7 & (41) & \\
\hline Regularly taken medications, $\mathrm{n}(\%)$ & & & & & & & 0.242 \\
\hline$<4$ & 24 & (17) & 14 & $(13)$ & 1 & (5) & \\
\hline $4-10$ & 91 & $(66)$ & 62 & (58) & 14 & (78) & \\
\hline over 10 & 24 & (17) & 31 & (28) & 3 & (17) & \\
\hline Diagnosis of memory disorder, $\mathrm{n}(\%)$ & 30 & $(22)$ & 45 & $(42)$ & 10 & (56) & $<0.001$ \\
\hline Independent mobility, n (\%) & 135 & (97) & 93 & (87) & 13 & (72) & $<0.001$ \\
\hline Living in own home, $\mathrm{n}(\%)$ & 117 & (84) & 65 & (61) & 12 & (67) & $<0.001$ \\
\hline Time to operation $<24 \mathrm{~h}, \mathrm{n}(\%)$ & 86 & $(63)$ & 57 & $(54)$ & 12 & $(67)$ & 0.296 \\
\hline NRS2002 median (IQR, range) & 2 & $(2-2 ; 1-5)$ & 2 & $(1-3 ; 1-5)$ & 3 & $(1.5-4.5 ; 2-5)$ & $<0.001$ \\
\hline
\end{tabular}

IQR=Interquartile range. Differences ( $p$-value) between groups were tested by Independent samples Kruskall-Wallis test or Pearson's chi-square test. Statistically significant $p$-values under 0.05 are presented in bold face. 
Table 2. Distribution of basic baseline characteristics according to NRS2002 (N=265)

\begin{tabular}{|c|c|c|c|c|c|c|c|}
\hline \multirow[b]{2}{*}{ Age, median (IQR, range) } & \multicolumn{2}{|c|}{$\begin{array}{c}\text { Normal/mild risk } \\
\mathrm{n}=198(75 \%)\end{array}$} & \multicolumn{2}{|c|}{$\begin{array}{c}\text { Moderate risk } \\
n=56(21 \%)\end{array}$} & \multicolumn{2}{|r|}{$\begin{array}{c}\text { Severe risk } \\
\mathrm{n}=11(4 \%)\end{array}$} & \multirow{2}{*}{$\begin{array}{l}p \\
0.652\end{array}$} \\
\hline & 85 & $(78-90 ; 65-103)$ & 85 & $(80-88 ; 65-98)$ & 80 & $(77-89 ; 67-93)$ & \\
\hline Women, $\mathrm{n}(\%)$ & 134 & (68) & 34 & (61) & 9 & (82) & 0.346 \\
\hline BMI, median (IQR, range) & 25 & $(23-28 ; 17-58)$ & 23 & $(20-26 ; 19-37)$ & 18 & $(16-19 ; 16-29)$ & $<0.001$ \\
\hline Alb, median (IQR, range) & 34 & $(31-36 ; 22-42)$ & 34 & $(31-37 ; 23-43)$ & 33 & $(27-37 ; 17-38)$ & 0.648 \\
\hline Fracture type, n (\%) & & & & & & & 0.192 \\
\hline Neck of femur & 119 & $(60)$ & 37 & (66) & 8 & (73) & \\
\hline Intertrochanteric & 70 & (35) & 13 & (23) & 2 & (18) & \\
\hline Subtrochanteric & 9 & (5) & 6 & (11) & 1 & (9) & \\
\hline ASA, $n(\%)$ & & & & & & & 0.920 \\
\hline $1-2$ & 27 & (14) & 7 & (13) & 1 & (9) & \\
\hline 3 & 128 & (65) & 40 & (71) & 7 & (64) & \\
\hline $4-5$ & 36 & (18) & 8 & (14) & 3 & (27) & \\
\hline Regularly taken medications, $\mathrm{n}(\%)$ & & & & & & & 0.242 \\
\hline$<4$ & 30 & $(15)$ & 8 & (14) & 1 & (9) & \\
\hline $4-10$ & 127 & (64) & 32 & (57) & 9 & (82) & \\
\hline over 10 & 41 & (21) & 16 & (29) & 1 & (9) & \\
\hline Diagnosis of memory disorder, $\mathrm{n}(\%)$ & 64 & (32) & 17 & (30) & 4 & (36) & 0.917 \\
\hline Independent mobility, $\mathrm{n}(\%)$ & 179 & (90) & 52 & (92) & 11 & $(100)$ & 1.000 \\
\hline Living in own home, $\mathrm{n}(\%)$ & 139 & (70) & 45 & $(80)$ & 10 & (91) & 0.547 \\
\hline Time to operation $<24 \mathrm{~h}, \mathrm{n}(\%)$ & 115 & (58) & 33 & (59) & 8 & (73) & 0.662 \\
\hline MNA-SF median (IQR, range) & 12 & $(11-13 ; 5-4)$ & 10 & $(9-11 ; 5-13)$ & 8 & $(6-10 ; 1-13)$ & $<0.001$ \\
\hline
\end{tabular}


Table 3. Patients' outcomes according to MNA-SF and NRS2002 (N=265).

\begin{tabular}{|c|c|c|c|c|c|c|c|}
\hline MNA-SF & Normal; $n=1$ & $9(52 \%)$ & Risk of malnutrit & $n ; n=108(41 \%)$ & Malnourished & $n=18(7 \%)$ & $\mathrm{p}$ \\
\hline Deceased within 1 month, $\mathrm{n}(\%)$ & 6 & $(4.3)$ & 8 & (7.4) & 6 & $(33.3)$ & 0.001 \\
\hline Deceased within 4 months, $\mathrm{n}(\%)$ & 14 & (10.1) & 23 & (21.3) & 10 & $(55.6)$ & $<0.001$ \\
\hline Readmissions within 1 month times, $\mathrm{n}(\%)$ & & & & & & & 0.005 \\
\hline 0 & 109 & (78.4) & 87 & $(80.6)$ & 9 & $(50.0)$ & \\
\hline 1 & 19 & $(13.7)$ & 8 & $(7.4)$ & 3 & $(16.7)$ & \\
\hline 2 or more & 5 & (3.6) & 3 & $(2.8)$ & 0 & $(0)$ & \\
\hline Deceased & 6 & (4.3) & 8 & (7.4) & 6 & (33.3) & \\
\hline Not known & 0 & (0) & 2 & (1.9) & 0 & (0) & \\
\hline 0 & 88 & $(63.3)$ & 57 & $(52.8)$ & 5 & $(27.8)$ & \\
\hline 1 & 18 & (12.9) & 14 & (13.0) & 2 & $(11.1)$ & \\
\hline 2 & 12 & $(8.6)$ & 3 & $(2.8)$ & 0 & (0) & \\
\hline 3 or more & 5 & (3.6) & 3 & (2.8) & 0 & (0) & \\
\hline Deceased & 15 & (10.8) & 23 & $(21.3)$ & 10 & $(55.6)$ & \\
\hline Not known & 1 & $(0.7)$ & 8 & $(7.4)$ & 1 & $(5.6)$ & \\
\hline LOS, days, median (range) & 22 & $(4-100)$ & 22 & $(3-129)$ & 24 & $(5-230)$ & 0.547 \\
\hline NRS2002 & Normal or mild ris & $n=198(75 \%)$ & Moderate risl & $n=56(21 \%)$ & Severe risk; & $=11(4 \%)$ & $\mathrm{p}$ \\
\hline Deceased within 4 months, $\mathrm{n}(\%)$ & 37 & (18.7) & 7 & (12.5) & 3 & $(27.3)$ & 0.382 \\
\hline Readmissions within 1 month times, $\mathrm{n}(\%)$ & & & & & & & 0.085 \\
\hline 0 & 157 & (79.3) & 42 & $(75.0)$ & 6 & $(54.5)$ & \\
\hline 1 & 21 & $(10.6)$ & 7 & $(12.5)$ & 2 & $(18.2)$ & \\
\hline 2 or more & 7 & (3.5) & 1 & $(1.8)$ & 0 & $(0)$ & \\
\hline Deceased & 13 & (6.6) & 5 & (8.9) & 2 & $(18.2)$ & \\
\hline Not known & 0 & (0) & 1 & (1.8) & 1 & $(9.1)$ & \\
\hline Readmissions within 4 months, $\mathrm{n}(\%)$ & & & & & & & 0.460 \\
\hline 0 & 113 & (57.1) & 33 & (58.9) & 4 & $(36.4)$ & \\
\hline 1 & 24 & (12.1) & 8 & (14.3) & 2 & $(18.2)$ & \\
\hline 2 & 11 & $(5.6)$ & 4 & (7.1) & 0 & (0) & \\
\hline 3 or more & 6 & (3.0) & 2 & $(3.6)$ & 0 & (0) & \\
\hline Deceased & 39 & $(19.2)$ & 7 & $(12.5)$ & 3 & $(27.3)$ & \\
\hline Not known & 6 & (3.0) & 2 & $(3.6)$ & 2 & $(18.2)$ & \\
\hline LOS, days, median (range) & 22 & $(3-135)$ & 23 & $(5-230)$ & 22 & $(5-114)$ & 0.614 \\
\hline
\end{tabular}

LOS= Length of stay in the central hospital and the primary care hospital. Differences between groups were tested by Fisher's exact test or Independent samples KruskallWallis test. Statistically significant $p$-values under 0.05 are presented in bold face. 